\title{
Linear Stability Conditions for a First Order $n$-Dimensional Mapping
}

\author{
Rafael Luís ${ }^{1,2}$
}

Received: 23 September 2020 / Accepted: 5 February 2021 / Published online: 25 February 2021

(c) The Author(s), under exclusive licence to Springer Nature Switzerland AG part of Springer Nature 2021

\begin{abstract}
In this paper we present an alternative way to compute the coefficients of a characteristic polynomial of a matrix via the trace, determinant and the sum of the minors that may be useful in determining the local stability conditions for mappings.
\end{abstract}

Keywords Characteristic polynomial $\cdot$ Minors $\cdot$ Difference equations $\cdot$ Local stability $\cdot$ Applications

Mathematics Subject Classification $37 \mathrm{C} 25 \cdot 37 \mathrm{C} 75 \cdot 39 \mathrm{~A} 30 \cdot 39 \mathrm{~A} 60$

\section{Introduction}

Stability is one of the most important concepts in dynamical systems. It is well known that, a fixed point of a discrete dynamical system is locally asymptotically stable if all the eigenvalues of the Jacobian matrix, evaluated at the fixed point, are less than one in absolute value.

However, in the most cases, we are not able to compute the eigenvalues. The challenge here is to provide stability conditions without knowing the eigenvalues of the Jacobian matrix.

The necessary and sufficient algebraic conditions for the roots of a real polynomial to lie inside the unit circle have been established by Jury [8] in a table form, where the constraints are obtained only by evaluation of second-order determinants.

In order to determine such a table, it is necessary to know all the coefficients of the characteristic polynomial of the Jacobian matrix. Those coefficients may be obtained from the trace, the determinant and the minors of the Jacobian matrix.

$凶$ Rafael Luís

rafael.luis.madeira@gmail.com

1 University of Madeira, Funchal, Madeira, Portugal

2 Center for Mathematical Analysis, Geometry, and Dynamical Systems, Technical University of Lisbon, Lisbon, Portugal

Birkhäuser 
In this paper, we present an alternative way to compute the values of the coefficients of a characteristic polynomial. We use some specific notation that turns such computation friendly, specifically from the computational point of view.

The tools that we present here, may be applied in stability theory of discrete dynamical systems. In the literature, there are conditions for 2-dimensional systems [6], 3-dimensional systems [2] and 4-dimensional systems [4] involving the trace, the determinant and the minors of the Jacobian matrix.

Beyond dimension 4, as far as we know, there are no studies for the linear stability conditions of discrete dynamical systems involving the trace, the determinant and the minors of the Jacobian matrix. These stability conditions may be determined from Jury's table.

The paper is organized as follows. In Sect. 2, we present the appropriate notation and the preliminary results for the rest of the paper. In Sect. 3, we revisit the characteristic polynomial of a matrix and determine the coefficients according to our needs. In Sect. 4 , we determine the necessary and sufficient conditions for local stability of a firstorder discrete dynamical system and in Sect. 5, we illustrate our results with concrete models, namely models in economics and in population dynamics.

\section{Preliminaries}

In this section we present some notations and preliminary results that we will use in the rest paper. We remark that some of the notations that we are going to follow may be used in the literature in a different way, but we are using it in this way since it seems more convenient to our results.

Let $A=\left[a_{i j}\right]$ be an $n \times n$ matrix with entries from $\mathbb{C}$, the set of complex numbers. By $\left|A_{i_{1}}\right|, i_{1}=1,2, \ldots, n$ we represent the $(n-1) \times(n-1)$ determinant of the $n$ sub-matrices $A_{i_{1}}$ obtained from $A$ by deleting the $i_{1}$ row and the $i_{1}$ column. Each one of those $n$ determinants represents precisely the $\left(i_{1}, i_{1}\right)$ - minor of $A$.

By $\left|A_{i_{1}, i_{2}}\right|, i_{1}, i_{2}=1,2, \ldots, n$, with $i_{1} \neq i_{2}$ we represent the $(n-2) \times(n-2)$ determinant of the sub-matrix $A_{i_{1}, i_{2}}$ obtained from $A$ by deleting the $i_{1}$ and $i_{2}$ rows and the $i_{1}$ and $i_{2}$ columns. And inductively, $\left|A_{i_{1}, \ldots, i_{k}}\right|, i_{1}, \ldots, i_{k}=1,2, \ldots, n$, with $i_{1} \neq i_{2} \neq \cdots \neq i_{k}$, is the $(n-k) \times(n-k)$ determinant of the sub-matrices $A_{i_{1}, \ldots, i_{k}}$ obtained from $A$ by deleting the $i_{1}, \ldots, i_{k}$ rows and the $i_{1}, \ldots, i_{k}$ columns.

For the sake of notation, we will represent the following sums by $\Delta_{j}, j=0,1,2, \ldots$. as follows:

$$
\begin{aligned}
\Delta_{0} & =|A| \\
\Delta_{1} & =\sum_{i_{1}=1}^{n}\left|A_{i_{1}}\right| \\
\Delta_{2} & =\sum_{i_{1}=1}^{n-1} \sum_{i_{2}=i_{1}+1}^{n}\left|A_{i_{1}, i_{2}}\right|=\sum_{1 \leq i_{1}<i_{2}}^{n-1, n}\left|A_{i_{1}, i_{2}}\right| \\
\vdots &
\end{aligned}
$$




$$
\Delta_{k}=\sum_{i_{1}=1}^{n-k+1} \sum_{i_{2}=i_{1}+1}^{n-k+2} \ldots \sum_{i_{k}=i_{k-1}+1}^{n}\left|A_{i_{1}, \ldots, i_{k}}\right|=\sum_{1 \leq i_{1}<\cdots<i_{k}}^{n-k+1, \ldots, n}\left|A_{i_{1}, \ldots, i_{k}}\right|
$$

\section{The Characteristic Equation Revisited}

Let $\lambda_{1}, \ldots, \lambda_{n}$ be the $n$ eigenvalues of an $n \times n$ matrix $A$. In [3] the author showed that the characteristic equation of $A$ can be given by

$$
(-1)^{n} x^{n}+(-1)^{n-1} p_{n-1} x^{n-1} \cdots-p_{3} x^{3}+p_{2} x^{2}-p_{1} x+p_{0}=0,
$$

where

$$
\begin{gathered}
p_{n-1}=\sum_{i_{1}=1}^{n} \lambda_{i_{1}}, \quad p_{n-2}=\sum_{1 \leq i_{1}<i_{2}}^{n-1, n} \lambda_{i_{1}} \lambda_{i_{2}}, \quad p_{n-3}=\sum_{1 \leq i_{1}<i_{2}<i_{3}}^{n-2, n-1, n} \lambda_{i_{1}} \lambda_{i_{2}} \lambda_{i_{3}}, \ldots, \\
p_{1}=\sum_{1 \leq i_{1}<\cdots<i_{n-1}}^{2, \ldots, n} \lambda_{i_{1}} \cdots \lambda_{i_{n-1}} \text { and } p_{0}=\sum_{1 \leq i_{1}<\cdots<i_{n}}^{1, \ldots, n} \lambda_{i_{1}} \cdots \lambda_{i_{n}}=\prod_{i=1}^{n} \lambda_{i}
\end{gathered}
$$

The values of $p_{i}, i=0,1, \ldots, n-1$, can be obtained via the numbers $\Delta_{i}$, defined in the previous section, namely $p_{i}=\Delta_{i}, i=0,1, \ldots, n-1$. This is precisely the following result:

Theorem 3.1 The characteristic equation (3.1) is equivalent to

$$
(-1)^{n} x^{n}+(-1)^{n-1} \Delta_{n-1} x^{n-1}+\cdots-\Delta_{3} x^{3}+\Delta_{2} x^{2}-\Delta_{1} x+\Delta_{0}=0 .
$$

Before we proceed to the proof of this result, we will illustrate it with an example. Consider the matrix

$$
A=\left(\begin{array}{ccccc}
-1 & 0 & 0 & 1 & -1 \\
1 & 0 & -1 & 1 & 0 \\
0 & -1 & 1 & 0 & -1 \\
-1 & -1 & 0 & 1 & 0 \\
0 & 1 & -1 & 0 & 1
\end{array}\right)
$$

The characteristic equation is given by $-x^{5}+2 x^{4}-x^{2}+6 x=0$. In order to compute the coefficients using the relations from the Sect. 2, one can proceed as follows:

$$
\begin{aligned}
& \Delta_{0}=|A|=0 \\
& \Delta_{1}=\sum_{i_{1}=1}^{5}\left|A_{i_{1}}\right|=\left|A_{1}\right|+\left|A_{2}\right|+\left|A_{3}\right|+\left|A_{4}\right|+\left|A_{5}\right|=0+0-4+0-2=-6 \\
& \Delta_{2}=\sum_{1 \leq i_{1}<i_{2}}^{4,5}\left|A_{i_{1}, i_{2}}\right|=\sum_{i_{2}=2}^{5}\left|A_{1, i_{2}}\right|+\sum_{i_{2}=3}^{5}\left|A_{2, i_{2}}\right|+\sum_{i_{2}=4}^{5}\left|A_{3, i_{2}}\right|+\sum_{i_{2}=5}^{5}\left|A_{4, i_{2}}\right|
\end{aligned}
$$




$$
\begin{aligned}
& =1+0-3+1=-1 \\
\Delta_{3} & =\sum_{1 \leq i_{1}<i_{2}<i_{3}}^{3,4,5}\left|A_{i_{1}, i_{2}, i_{3}}\right|=\sum_{2 \leq i_{2}<i_{3}}^{4,5}\left|A_{1, i_{2}, i_{3}}\right|+\sum_{3 \leq i_{2}<i_{3}}^{4,5}\left|A_{2, i_{2}, i_{3}}\right|+\sum_{4 \leq i_{2}<i_{3}}^{4,5}\left|A_{3, i_{2}, i_{3}}\right|=\cdots=0 \\
\Delta_{4} & =\sum_{1 \leq i_{1}<\cdots<i_{4}}^{2,3,4,5}\left|A_{i_{1}, \ldots, i_{4}}\right|=\left|A_{1,2,3,4}\right|+\left|A_{1,2,3,5}\right|+\left|A_{1,2,4,5}\right|+\left|A_{1,3,4,5}\right|+\left|A_{2,3,4,5}\right| \\
& =1+1+1+0-1=2
\end{aligned}
$$

In this example, the set of eigenvalues of $A$ is given by

$$
\left\{\lambda_{1}, \ldots, \lambda_{5}\right\}=\left\{\frac{1}{2}(1+\sqrt{13}), \frac{1}{2}(1+i \sqrt{7}), \frac{1}{2}(1-i \sqrt{7}), \frac{1}{2}(1-\sqrt{13}), 0\right\} .
$$

Clearly, $p_{0}=0$ and

$$
\begin{aligned}
& p_{1}=\sum_{1 \leq i_{1}<\cdots<i_{4}}^{2, \ldots, 5} \lambda_{i_{1}} \cdots \lambda_{i_{4}}=\lambda_{1} \cdots \lambda_{4}+0=-6, \\
& p_{2}=\sum_{1 \leq i_{1}<\cdots<i_{3}}^{3,4,5} \lambda_{i_{1}} \lambda_{i_{2}} \lambda_{i_{3}}=\lambda_{1} \lambda_{2} \lambda_{3}+\lambda_{1} \lambda_{2} \lambda_{4}+\lambda_{1} \lambda_{3} \lambda_{4}+\lambda_{2} \lambda_{3} \lambda_{4}+0=-1, \\
& p_{3}=\sum_{1 \leq i_{1}<i_{2}}^{4,5} \lambda_{i_{1}} \lambda_{i_{2}}=\lambda_{1} \lambda_{2}+\lambda_{1} \lambda_{3}+\cdots+\lambda_{4} \lambda_{5}=0,
\end{aligned}
$$

and

$$
p_{4}=\sum_{i_{1}=1}^{5} \lambda_{i_{1}}=\lambda_{1}+\cdots+\lambda_{5}=2
$$

We now present the proof of Theorem 3.1.

Proof The proof is given by mathematical induction for $n \geq 2$. The base case establish that the characteristic equation of a $2 \times 2$ matrix $A$ is given by

$$
x^{2}-\Delta_{1} x+\Delta_{0}=x^{2}-\operatorname{tr}(A) x+|A|=0
$$

where $t r$ is the trace of the matrix $A$, a well known relation in the literature (see for instance [6,pp. 200]). The cases when $n=3$ and $n=4$ may be found in Brooks [2,4], respectively.

Let us assume in the induction hypothesis that the characteristic equation of an $n \times n$ matrix $A$ is given by 


$$
\begin{aligned}
& (-1)^{n} x^{n}+(-1)^{n-1} \sum_{1 \leq i_{1}<\cdots<i_{n-1}}^{2, \ldots, n}\left|A_{i_{1}, \ldots, i_{n-1}}\right| x^{n-1} \\
& +(-1)^{n-2} \sum_{1 \leq i_{1}<\cdots<i_{n-2}}^{3, \ldots, n}\left|A_{i_{1}, \ldots, i_{n-2}}\right| x^{n-2}+\cdots+\sum_{1 \leq i_{1}<i_{2}}^{n-1, n}\left|A_{i_{1}, i_{2}}\right| x^{2}-\sum_{i_{1}=1}^{n}\left|A_{i_{1}}\right| x+|A|=0 .
\end{aligned}
$$

In order to show the inductive step, we start by writing the characteristic equation of an $(n+1) \times(n+1)$ matrix $A$, i.e. $\left|A-x I_{n+1}\right|=0$, or equivalently,

$$
\left|\begin{array}{cccc}
a_{11}-x & a_{12} & \ldots & a_{1 n+1} \\
a_{21} & a_{22}-x & \ldots & a_{2 n+1} \\
\vdots & & \ddots & \\
a_{n+11} & a_{n+12} & \ldots & a_{n+1 n+1}-x
\end{array}\right|=0
$$

Applying the properties of determinant in the last column, we get

$$
\begin{gathered}
\left|\begin{array}{ccccc}
a_{11}-x & a_{12} & \ldots & a_{1 n} & a_{1 n+1} \\
a_{21} & a_{22}-x & \ldots & a_{2 n} & a_{2 n+1} \\
\vdots & & \ddots & & \\
a_{n 1} & a_{n 2} & \ldots & a_{n n}-x & a_{n n+1} \\
a_{n+11} & a_{n+12} & \ldots & a_{n+1 n} & a_{n+1 n+1}
\end{array}\right| \\
-x\left|\begin{array}{cccc}
a_{11}-x & a_{12} & \ldots & a_{1 n} \\
a_{21} & a_{22}-x & \ldots & a_{2 n} \\
\vdots & & \ddots & \\
a_{n 1} & a_{n 2} & \ldots & a_{n n}-x
\end{array}\right|=0 .
\end{gathered}
$$

Regarding to the $(n+1) \times(n+1)$ determinant of (3.4), we apply successively, in each column, the illustrated property and we obtain

$$
\begin{aligned}
& \left|\begin{array}{cccc}
a_{11}-x & a_{12} & \ldots & a_{1 n+1} \\
a_{21} & a_{22}-x & \ldots & a_{2 n+1} \\
\vdots & \ddots & \\
a_{n+11} & a_{n+12} & \ldots & a_{n+1 n+1}
\end{array}\right|=|A|-\sum_{i_{1}=1}^{n}\left|A_{i_{1}}\right| x+\sum_{1 \leq i_{1}<i_{2}}^{n-1, n}\left|A_{i_{1} i_{2}}\right| x^{2}-\sum_{1 \leq i_{1}<i_{2}<i_{3}}^{n-2, n-1, n}\left|A_{i_{1} i_{2} i_{3}}\right| x^{3} \\
& +\cdots+(-1)^{n-1} \sum_{1 \leq i_{1}<\cdots<i_{n-1}}^{2, \ldots, n}\left|A_{i_{1}, \ldots, i_{n-1}}\right| x^{n-1}+(-1)^{n} \sum_{1 \leq i_{1}<\cdots<i_{n}}^{1, \ldots, n}\left|A_{i_{1}, \ldots, i_{n}}\right| x^{n} .
\end{aligned}
$$

Now, relating to the $n \times n$ determinant of (3.4), we apply the induction hypothesis (3.3) and get 


$$
\begin{aligned}
& -x\left|\begin{array}{cccc}
a_{11}-x & a_{12} & \ldots & a_{1 n} \\
a_{21} & a_{22}-x & \ldots & a_{2 n} \\
\vdots & & \ddots & \\
a_{n 1} & a_{n 2} & \ldots & a_{n n}-x
\end{array}\right|=-x\left((-1)^{n} x^{n}+(-1)^{n-1} \sum_{1 \leq i_{1}<\ldots<i_{n-1}}^{2, \ldots, n}\left|A_{i_{1}, \ldots, i_{n-1}}\right| x^{n-1}\right. \\
& \left.+(-1)^{n-2} \sum_{1 \leq i_{1}<\ldots<i_{n-2}}^{3, \ldots, n}\left|A_{i_{1}, \ldots, i_{n-2}}\right| x^{n-2}+\cdots+\sum_{1 \leq i_{1}<i_{2}}^{n-1, n}\left|A_{i_{1}, i_{2}}\right| x^{2}-\sum_{i_{1}=1}^{n}\left|A_{i_{1}}\right| x+|A|\right) .
\end{aligned}
$$

Since in (3.6) one can write $|A|=\left|A_{n+1}\right|,\left|A_{i}\right|=\left|A_{i, n+1}\right|$, etc, it follows that (3.6) is equivalent to

$$
-x\left|\begin{array}{cccc}
a_{11}-x & a_{12} & \ldots & a_{1 n} \\
a_{21} & a_{22}-x & \ldots & a_{2 n} \\
\vdots & & \ddots & \\
a_{n 1} & a_{n 2} & \ldots & a_{n n}-x
\end{array}\right|=(-1)^{n+1} x^{n+1}
$$

$$
\begin{aligned}
& +(-1)^{n} \sum_{1 \leq i_{1}<\cdots<i_{n-1}}^{2, \ldots, n}\left|A_{i_{1}, \ldots, i_{n-1}, n+1}\right| x^{n}+(-1)^{n-1} \sum_{1 \leq i_{1}<\cdots<i_{n-2}}^{3, \ldots, n}\left|A_{i_{1}, \ldots, i_{n-2}, n+1}\right| x^{n-1} \\
& +\cdots-\sum_{1 \leq i_{1}<i_{2}}^{n-1, n}\left|A_{i_{1}, i_{2}, n+1}\right| x^{3}+\sum_{i_{1}=1}^{n}\left|A_{i_{1}, n+1}\right| x^{2}-\left|A_{n+1}\right| x .
\end{aligned}
$$

Now, adding (3.5) and (3.7), the equation (3.4) is equivalent to

$$
\begin{aligned}
& (-1)^{n+1} x^{n+1}+(-1)^{n}\left(\sum_{1 \leq i_{1}<\ldots<i_{n-1}}^{2, \ldots, n}\left|A_{i_{1}, \ldots, i_{n-1}, n+1}\right|+\sum_{1 \leq i_{1}<\ldots<i_{n}}^{1, \ldots, n}\left|A_{i_{1}, \ldots, i_{n}}\right|\right) x^{n} \\
& +(-1)^{n-1}\left(\sum_{1 \leq i_{1}<\ldots<i_{n-2}}^{3, \ldots, n}\left|A_{i_{1}, \ldots, i_{n-2}, n+1}\right|+\sum_{1 \leq i_{1}<\ldots<i_{n-1}}^{2, \ldots, n}\left|A_{i_{1}, \ldots, i_{n-1}}\right|\right) x^{n-1} \\
& +\cdots-\left(\sum_{1 \leq i_{1}<i_{2}}^{n-1, n}\left|A_{i_{1}, i_{2}, n+1}\right|+\sum_{1 \leq i_{1}<i_{2}<i_{3}}^{n-2, n-1, n}\left|A_{i_{1} i_{2} i_{3}}\right|\right) x^{3} \\
& +\left(\sum_{i_{1}=1}^{n}\left|A_{i_{1}, n+1}\right|+\sum_{1 \leq i_{1}<i_{2}}^{n-1, n}\left|A_{i_{1} i_{2}}\right|\right) x^{2}-\left(\left|A_{n+1}\right|+\sum_{i_{1}=1}^{n}\left|A_{i_{1}}\right|\right) x+|A|=0 .
\end{aligned}
$$

Simplifying we obtain

$$
\begin{aligned}
& (-1)^{n+1} x^{n+1}+(-1)^{n} \sum_{1 \leq i_{1}<\cdots<i_{n}}^{2, \ldots, n+1}\left|A_{i_{1}, \ldots, i_{n}}\right| x^{n}+(-1)^{n-1} \sum_{1 \leq i_{1}<\cdots<i_{n-1}}^{3, \ldots, n+1} \\
& \left|A_{i_{1}, \ldots, i_{n-1}}\right| x^{n-1}+\cdots-\sum_{1 \leq i_{1}<i_{2}<i_{3}}^{n-1, n, n+1}\left|A_{i_{1} i_{2} i_{3}}\right| x^{3}
\end{aligned}
$$




$$
+\sum_{1 \leq i_{1}<i_{2}}^{n, n+1}\left|A_{i_{1} i_{2}}\right| x^{2}-\sum_{i_{1}=1}^{n+1}\left|A_{i_{1}}\right| x+|A|=0
$$

or equivalently,

$$
\begin{aligned}
& (-1)^{n+1} x^{n+1}+(-1)^{n} \Delta_{n} x^{n}+(-1)^{n-1} \Delta_{n-1} x^{n-1} \cdots-\Delta_{3} x^{3} \\
& +\Delta_{2} x^{2}-\Delta_{1} x+\Delta_{0}=0 .
\end{aligned}
$$

\section{Applications: Local Stability of Mappings}

It is well known that, a fixed point $\mathbf{x}^{*}$ of a discrete dynamical system of the form $\mathbf{x}_{n+1}=G\left(\mathbf{x}_{n}\right)$, for $n=0,1,2 \ldots$, where $G: \mathbb{R}^{n} \rightarrow \mathbb{R}^{n}$ is a $C^{k}$ smooth mapping, for some $k \geq 1$, is locally asymptotically stable if all the eigenvalues of the matrix $A=J G\left(\mathbf{x}^{*}\right)$ are less than one in absolute value, where $J G$ is the Jacobian matrix of the function $G$, evaluated at the fixed point $\mathbf{x}^{*}$. The fixed point $\mathbf{x}^{*}$ is unstable if there exists at least one of the eigenvalues of $A$ with absolute value greater than one.

So, in order to study the stability of a fixed point in a discrete dynamical system, we just need to study the roots of the characteristic polynomial of the Jacobian matrix evaluated at the fixed point.

In [8], Jury established necessary and sufficient algebraic conditions for the roots of a real polynomial to lie inside the unit circle. Those conditions are given in table form where the constraints are obtained only by evaluating second-order determinants. The usefulness of Jury's table may be found in those cases where the values of the coefficients of the real polynomial are known.

This algebraic test, similar in form to Routh's table [14], consists of two parts: (i) simple test for necessary conditions and (ii) test for sufficient conditions.

For a polynomial of the form

$$
f(x)=a_{n} x^{n}+a_{n-1} x^{n-1}+\cdots+a_{2} x^{2}+a_{1} x+a_{0}=0, a_{n}>0,
$$

the necessary conditions for stability are

$$
f(1)>0 \text { and }(-1)^{n} f(-1)>0 .
$$

In order to establish the sufficient conditions we need to construct Table 1, where

$$
\begin{aligned}
b_{k} & =\left|\begin{array}{cc}
a_{0} & a_{n-k} \\
a_{n} & a_{k}
\end{array}\right|, k=0,1,2, \ldots, n-1, \\
c_{k} & =\left|\begin{array}{cc}
b_{0} & b_{n-1-k} \\
b_{n-1} & b_{k}
\end{array}\right|, k=0,1,2, \ldots, n-2, \\
d_{k} & =\left|\begin{array}{cc}
c_{0} & c_{n-2-k} \\
c_{n-2} & c_{k}
\end{array}\right|, k=0,1,2, \ldots, n-3,
\end{aligned}
$$


Table 1 Jury's table

\begin{tabular}{lllllllll}
\hline Row & $x^{0}$ & $x^{1}$ & $x^{2}$ & $\ldots$ & $x^{n-k}$ & $\ldots$ & $x^{n-1}$ & $x^{n}$ \\
\hline 1 & $a_{0}$ & $a_{1}$ & $a_{2}$ & $\ldots$ & $a_{n-k}$ & $\ldots$ & $a_{n-1}$ & $a_{n}$ \\
2 & $a_{n}$ & $a_{n-1}$ & $a_{n-2}$ & $\ldots$ & $a_{k}$ & $\ldots$ & $a_{1}$ & $a_{0}$ \\
3 & $b_{0}$ & $b_{1}$ & $b_{2}$ & $\ldots$ & $b_{n-k}$ & $\ldots$ & $b_{n-1}$ & - \\
4 & $b_{n-1}$ & $b_{n-2}$ & $b_{n-3}$ & $\ldots$ & $b_{k}$ & $\ldots$ & $b_{0}$ & - \\
5 & $c_{0}$ & $c_{1}$ & $c_{2}$ & $\ldots$ & $\ldots$ & $c_{n-2}$ & - & - \\
6 & $c_{n-2}$ & $c_{n-3}$ & $c_{n-4}$ & $\ldots$ & $\ldots$ & $c_{0}$ & - & - \\
$\vdots$ & & & & & & & & \\
$2 n-5$ & $p_{0}$ & $p_{1}$ & $p_{2}$ & $p_{3}$ & - & $\ldots$ & - & - \\
$2 n-4$ & $p_{3}$ & $p_{2}$ & $p_{1}$ & $p_{0}$ & - & $\ldots$ & - & - \\
$2 n-3$ & $q_{0}$ & $q_{1}$ & $q_{2}$ & - & $\ldots$ & $\ldots$ & - & - \\
\hline
\end{tabular}

etc.

The sufficient conditions for stability are the following $n-1$ relations:

$$
\left|a_{0}\right|<a_{n}, \quad\left|b_{0}\right|>\left|b_{n-1}\right|, \quad\left|c_{0}\right|>\left|c_{n-2}\right|, \ldots,\left|p_{0}\right|>\left|p_{3}\right|, \quad\left|q_{0}\right|>\left|q_{2}\right| .
$$

From now on, we assume that $A$ is the Jacobian matrix, evaluated at a fixed point $\mathbf{x}^{*}$ of a discrete dynamical system of the form $\mathbf{x}_{n+1}=G\left(\mathbf{x}_{n}\right)$, for $n=0,1,2 \ldots$ and the characteristic equation of $A$ is given by (3.1).

\subsection{Two-dimensional Maps}

The characteristic equation of a $2 \times 2$ Jacobian matrix $A$, evaluated at the fixed point, is given by $f(x)=x^{2}-\Delta_{1} x+\Delta_{0}=0$. The necessary conditions are $f(1)>0$ and $f(-1)>0$, i.e. $\Delta_{0}>\Delta_{1}-1$ and $\Delta_{0}>-\left(\Delta_{1}+1\right)$, or equivalently $\left|\Delta_{1}\right|<1+\Delta_{0}$. The sufficient condition is $\left|\Delta_{0}\right|<1$. In other words, the necessary and sufficient conditions for a fixed point to be locally stable are

$$
\operatorname{det}(A)>\operatorname{tr}(A)-1, \quad \operatorname{det}(A)>-\operatorname{tr}(A)-1, \quad \operatorname{det}(A)<1 .
$$

Notice that the first two inequalities together implies that $\operatorname{det}(A)>-1$. This set of inequalities, generates the well known triangle of stability, in the trace-determinant plane (see Figure 4.10 in [6]).

\subsection{Three-dimensional Maps}

The characteristic equation of a $3 \times 3$ Jacobian matrix $A$, evaluated at the fixed point, is given by $f(x)=x^{3}-\Delta_{2} x^{2}+\Delta_{1} x-\Delta_{0}=0$. The necessary conditions are

$$
-\left(1+\Delta_{1}\right)<\Delta_{2}+\Delta_{0}<1+\Delta_{1}
$$


The Jury's table for this equation is

\begin{tabular}{lllll}
\hline Row & $x^{0}$ & $x^{1}$ & $x^{2}$ & $x^{3}$ \\
\hline 1 & $-\Delta_{0}$ & $\Delta_{1}$ & $-\Delta_{2}$ & 1 \\
2 & 1 & $-\Delta_{2}$ & $\Delta_{1}$ & $-\Delta_{0}$ \\
3 & $\Delta_{0}^{2}-1$ & $-\Delta_{0} \Delta_{1}+\Delta_{2}$ & $\Delta_{0} \Delta_{2}-\Delta_{1}$ & - \\
\hline
\end{tabular}

Hence, the sufficient conditions are $\left|-\Delta_{0}\right|<1$ and $\left|\Delta_{0}^{2}-1\right|>\left|\Delta_{0} \Delta_{2}-\Delta_{1}\right|$, i.e., $\left|\Delta_{0}\right|<1$ and $\left|\Delta_{0} \Delta_{2}-\Delta_{1}\right|<1-\Delta_{0}^{2}$.

Consequently, the necessary and sufficient conditions for local stability of three dimensional system are

$$
\left|\Delta_{2}+\Delta_{0}\right|<1+\Delta_{1},\left|\Delta_{0}\right|<1,\left|\Delta_{0} \Delta_{2}-\Delta_{1}\right|<1-\Delta_{0}^{2}
$$

Remark 4.1 Notice that, if $\Delta_{0}=0$ (i.e., if one of the eigenvalues of the Jacobian matrix is zero), then those conditions are

$$
\left|\Delta_{2}\right|<1+\Delta_{1}, \quad\left|\Delta_{1}\right|<1
$$

which are similar to the case of $n=2$ by replacing $\Delta_{i}$ to $\Delta_{i-1}, i=1,2$.

\subsection{Four-dimensional Maps}

The characteristic equation of a $4 \times 4$ Jacobian matrix $A$, evaluated at the fixed point, is given by $f(x)=x^{4}-\Delta_{3} x^{3}+\Delta_{2} x^{2}-\Delta_{1} x+\Delta_{0}=0$. The necessary conditions are

$$
\left|\Delta_{3}+\Delta_{1}\right|<1+\Delta_{2}+\Delta_{0}
$$

The Jury's table for this equation is

\begin{tabular}{llllll}
\hline Row & $x^{0}$ & $x^{1}$ & $x^{2}$ & $x^{3}$ & $x^{4}$ \\
\hline 1 & $\Delta_{0}$ & $-\Delta_{1}$ & $\Delta_{2}$ & $-\Delta_{3}$ & 1 \\
2 & 1 & $-\Delta_{3}$ & $\Delta_{2}$ & $-\Delta_{1}$ & $\Delta_{0}$ \\
3 & $\Delta_{0}^{2}-1$ & $\Delta_{3}-\Delta_{0} \Delta_{1}$ & $\Delta_{2}\left(\Delta_{0}-1\right)$ & $\Delta_{1}-\Delta_{0} \Delta_{3}$ & - \\
4 & $\Delta_{1}-\Delta_{0} \Delta_{3}$ & $\Delta_{2}\left(\Delta_{0}-1\right)$ & $\Delta_{3}-\Delta_{0} \Delta_{1}$ & $\Delta_{0}^{2}-1$ & - \\
5 & $c_{0}$ & $c_{1}$ & $c_{2}$ & - & - \\
\hline
\end{tabular}

where

$$
c_{0}=\left(\Delta_{0}^{2}-1\right)^{2}-\left(\Delta_{1}-\Delta_{0} \Delta_{3}\right)^{2}
$$




$$
\begin{aligned}
& c_{1}=\left(\Delta_{0}^{2}-1\right)\left(\Delta_{3}-\Delta_{0} \Delta_{1}\right)-\Delta_{2}\left(\Delta_{0}-1\right)\left(\Delta_{1}-\Delta_{0} \Delta_{3}\right), \\
& c_{2}=\left(\Delta_{0}^{2}-1\right) \Delta_{2}\left(\Delta_{0}-1\right)-\left(\Delta_{3}-\Delta_{0} \Delta_{1}\right)\left(\Delta_{1}-\Delta_{0} \Delta_{3}\right) .
\end{aligned}
$$

The sufficient conditions are $\left|\Delta_{0}\right|<1,\left|\Delta_{0}^{2}-1\right|>\left|\Delta_{1}-\Delta_{0} \Delta_{3}\right|$ and $\left|c_{0}\right|>\left|c_{2}\right|$.

Simplifying, we obtain that the necessary and sufficient conditions are

$$
\begin{aligned}
& \left|\Delta_{3}+\Delta_{1}\right|<1+\Delta_{2}+\Delta_{0}, \\
& \left|\Delta_{0}\right|<1, \quad\left|\Delta_{1}-\Delta_{0} \Delta_{3}\right|<1-\Delta_{0}^{2}, \quad\left|c_{0}\right|>\left|c_{2}\right| .
\end{aligned}
$$

Remark 4.2 1. Notice that, if $\Delta_{0}=0$ (i.e., if one of the eigenvalues of the Jacobian matrix is zero), then those conditions are equivalent (after simplifying) to

$$
\left|\Delta_{3}+\Delta_{1}\right|<1+\Delta_{2}, \quad\left|\Delta_{1}\right|<1,\left|\Delta_{2}-\Delta_{1} \Delta_{3}\right|<1-\Delta_{1}^{2}
$$

which are similar to the case of $n=3$ by replacing $\Delta_{i}$ to $\Delta_{i-1}, i=1,2,3$.

2. In the case that $\Delta_{0}=0$ and $\Delta_{1}=0$ (i.e., if the Jacobian matrix has an eigenvalue zero with algebraic multiplicity 2 ), then those conditions are equivalent (after simplifying) to

$$
\left|\Delta_{3}\right|<1+\Delta_{2}, \quad\left|\Delta_{2}\right|<1
$$

which are similar to the case of $n=2$ by replacing $\Delta_{i}$ to $\Delta_{i-2}, i=2,3$.

\subsection{Five-dimensional Maps}

The characteristic equation of a $5 \times 5$ Jacobian matrix $A$, evaluated at the fixed point, is given by $f(x)=x^{5}-\Delta_{4} x^{4}+\Delta_{3} x^{3}-\Delta_{2} x^{2}+\Delta_{1} x-\Delta_{0}=0$. The necessary conditions are

$$
\left|\Delta_{4}+\Delta_{2}+\Delta_{0}\right|<1+\Delta_{3}+\Delta_{1}
$$

The Jury's table for this equation is

\begin{tabular}{lllllll}
\hline Row & $x^{0}$ & $x^{1}$ & $x^{2}$ & $x^{3}$ & $x^{4}$ & $x^{5}$ \\
\hline 1 & $-\Delta_{0}$ & $\Delta_{1}$ & $-\Delta_{2}$ & $\Delta_{3}$ & $-\Delta_{4}$ & 1 \\
2 & 1 & $-\Delta_{4}$ & $\Delta_{3}$ & $-\Delta_{2}$ & $\Delta_{1}$ & $-\Delta_{0}$ \\
3 & $\Delta_{0}^{2}-1$ & $-\left(\Delta_{4}+\Delta_{0} \Delta_{1}\right)$ & $\Delta_{2} \Delta_{0}-\Delta_{3}$ & $\Delta_{1}-\Delta_{0} \Delta_{3}$ & $\Delta_{4} \Delta_{0}-\Delta_{1}$ & - \\
4 & $\Delta_{4} \Delta_{0}-\Delta_{1}$ & $\Delta_{1}-\Delta_{0} \Delta_{3}$ & $\Delta_{2} \Delta_{0}-\Delta_{3}$ & $-\left(\Delta_{4}+\Delta_{0} \Delta_{1}\right)$ & $\Delta_{0}^{2}-1$ & - \\
5 & $c_{0}$ & $c_{1}$ & $c_{2}$ & $c_{3}$ & - & \\
6 & $c_{3}$ & $c_{2}$ & $c_{1}$ & $c_{0}$ & - & \\
7 & $d_{0}$ & $d_{1}$ & $d_{2}$ & - & - & \\
\hline
\end{tabular}


where

$$
\begin{aligned}
& c_{0}=\left(\Delta_{0}^{2}-1\right)^{2}-\left(\Delta_{4} \Delta_{0}-\Delta_{1}\right)^{2}, \\
& c_{1}=\left(\Delta_{1}-\Delta_{0} \Delta_{3}\right)\left(\Delta_{1}-\Delta_{0} \Delta_{4}\right)-\left(\Delta_{0}^{2}-1\right)\left(\Delta_{0} \Delta_{1}+\Delta_{4}\right), \\
& c_{2}=\left(\Delta_{0} \Delta_{2}-\Delta_{3}\right)\left(\Delta_{1}+\Delta_{0}^{2}-\Delta_{0} \Delta_{4}-1\right), \\
& c_{3}=\left(\Delta_{1}-\Delta_{0} \Delta_{3}\right)\left(\Delta_{0}^{2}-1\right)-\left(\Delta_{4}+\Delta_{0} \Delta_{1}\right)\left(\Delta_{4} \Delta_{0}-\Delta_{1}\right),
\end{aligned}
$$

and $d_{0}=c_{0}^{2}-c_{3}^{2}$ and $d_{2}=c_{2} c_{0}-c_{1} c_{3}$.

The sufficient conditions are $\left|\Delta_{0}\right|<1,\left|\Delta_{0}^{2}-1\right|>\left|\Delta_{4} \Delta_{0}-\Delta_{1}\right|,\left|c_{0}\right|>\left|c_{3}\right|$ and $\left|d_{0}\right|>\left|d_{3}\right|$.

Simplifying, we obtain that the necessary and sufficient conditions are

$$
\begin{aligned}
& \left|\Delta_{3}+\Delta_{1}\right|<1+\Delta_{2}+\Delta_{0}, \\
& \left|\Delta_{0}\right|<1, \quad\left|\Delta_{4} \Delta_{0}-\Delta_{1}\right|<1-\Delta_{0}^{2}, \\
& \left|c_{0}\right|>\left|c_{3}\right|, \quad\left|d_{0}\right|>\left|d_{2}\right| .
\end{aligned}
$$

Remark 4.3 1. Notice that, if $\Delta_{0}=0$ (i.e., if one of the eigenvalues of the Jacobian matrix is zero), then those conditions are similar to the case of $n=4$ by replacing $\Delta_{i}$ to $\Delta_{i-1}, i=1,2,3,4$.

2. In the case that $\Delta_{0}=0$ and $\Delta_{1}=0$ (i.e., if the Jacobian matrix has an eigenvalue zero with algebraic multiplicity 2 ), then these conditions are similar to the case of $n=3$ by replacing $\Delta_{i}$ to $\Delta_{i-2}, i=2,3,4$.

3. In the case that $\Delta_{0}=0, \Delta_{1}=0$, and $\Delta_{2}=0$ (i.e., if the Jacobian matrix has an eigenvalue zero with algebraic multiplicity 3 ), then those conditions are similar to the case of $n=2$ by replacing $\Delta_{i}$ to $\Delta_{i-3}, i=3,4$.

\section{5 n-dimensional Maps}

As it is clear in the previous subsections, as the order of the system increases the computations become more complicated, long and the algebraic manipulations are not feasible. However, one can see that the necessary conditions for an $n$-dimensional system are

$$
\left\{\begin{array}{l}
\left|\sum_{i=1}^{p} \Delta_{2 i-1}\right|<1+\sum_{i=0}^{p-1} \Delta_{2 i} \text { if } n=2 p \\
\left|\sum_{i=0}^{p} \Delta_{2 i}\right|<1+\sum_{i=1}^{p} \Delta_{2 i-1} \text { if } n=2 p+1
\end{array},\right.
$$

and the two following sufficient conditions

$$
\left|\Delta_{0}\right|<1, \quad\left|\Delta_{0} \Delta_{n-1}-\Delta_{1}\right|<1-\Delta_{0}^{2}
$$

The missing $n-4$ sufficient conditions are obtained from Jury's table. 


\section{Illustrative Examples}

In this section we illustrate the previous results in certain models that are parameter dependent, i.e, we determine the region, whenever possible, in the parameter space, where the respective fixed points are locally stable.

\subsection{Cournot Duopoly Game Model}

In [1] a Cournot duopoly game model is studied. The model is given by

$$
\left\{\begin{array}{l}
z_{1}(n+1)=z_{1}(n)\left(1+v_{1}\left(A-c_{1}\right)-2 B v_{1} z_{1}(n)-B v_{1} z_{2}(n)\right) \\
z_{2}(n+1)=z_{2}(n)\left(1+v_{2}\left(A-c_{2}\right)-2 B v_{2} z_{2}(n)-B v_{2} z_{1}(n)\right)
\end{array},\right.
$$

where $z_{1}(n)$ and $z_{2}(n)$ represents the production of the firms $z_{1}$ and $z_{2}$ at time period $n, n=0,1,2, \ldots$, respectively, $v_{i}>0, i=1,2$ is the relative speed of production adjustment of producer $i, c_{i}, i=1,2$ is the marginal cost and $A, B>0$ are the demand parameters.

The local properties of the positive equilibrium point of Model (5.1) as well as the dynamics of the critical curves are well explained and detailed studied.

We will now make a change of variables in Model (5.1) in order to reduce the number of parameters and make its study more friendly.

Let $z_{i}(n)=\frac{1+v_{i}\left(A-c_{i}\right)}{2 B v_{i}} x_{i}(n)$, for $i=1,2$. Substituting in the first equation of (5.1) we get

$$
\begin{aligned}
\frac{1+v_{1}\left(A-c_{1}\right)}{2 B v_{1}} x_{1}(n+1)= & \frac{1+v_{1}\left(A-c_{1}\right)}{2 B v_{1}} x_{1}(n)\left[1+v_{1}\left(A-c_{1}\right)\right. \\
& \left.-2 B v_{1} \frac{1+v_{1}\left(A-c_{1}\right)}{2 B v_{1}} x_{1}(n)-B v_{1} \frac{1+v_{2}\left(A-c_{2}\right)}{2 B v_{2}} x_{2}(n)\right],
\end{aligned}
$$

or equivalently,

$x_{1}(n+1)=\left(1+v_{1}\left(A-c_{1}\right)\right)\left(x_{1}(n)-x_{1}^{2}(n)\right)-\frac{v_{1}}{2 v_{2}}\left(1+v_{2}\left(A-c_{2}\right)\right) x_{2}(n) x_{1}(n)$.

Analogously, we get

$x_{2}(n+1)=\left(1+v_{2}\left(A-c_{2}\right)\right)\left(x_{2}(n)-x_{2}^{2}(n)\right)-\frac{v_{2}}{2 v_{1}}\left(1+v_{1}\left(A-c_{1}\right)\right) x_{1}(n) x_{2}(n)$.

By letting $a=1+v_{1}\left(A-c_{1}\right), b=1+v_{2}\left(A-c_{2}\right), c=\frac{v_{1}\left(1+v_{2}\left(A-c_{2}\right)\right)}{2 v_{2}\left(1+v_{1}\left(A-c_{1}\right)\right)}$ and $d=\frac{v_{2}\left(1+v_{1}\left(A-c_{1}\right)\right)}{2 v_{1}\left(1+v_{2}\left(A-c_{2}\right)\right)}$, we obtain

$$
\left\{\begin{array}{l}
x_{1}(n+1)=a x_{1}(n)\left(1-x_{1}(n)-c x_{2}(n)\right) \\
x_{2}(n+1)=b x_{2}(n)\left(1-x_{2}(n)-d x_{1}(n)\right)
\end{array} .\right.
$$


Fig. 1 A prototype of the the domain $\mathbf{D}$ of the Model (5.2). In this example the values of the parameters are $a=2, b=2$, $c=0.5$ and $d=0.5$

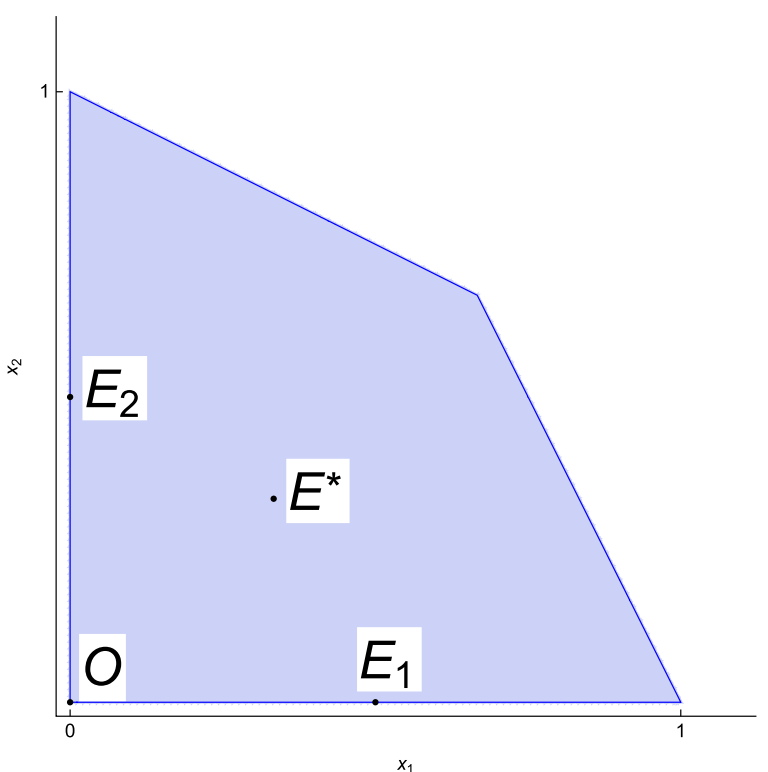

This new modified Cournot duopoly game model has the following fixed points: the origin $\mathbf{O}$, two fixed points on the axes $\mathbf{E}_{1}=\left(\frac{a-1}{a}, 0\right)$ and $\mathbf{E}_{2}=\left(0, \frac{b-1}{b}\right)$ and a positive fixed point $\mathbf{E}^{*}=\left(\frac{a[c+b(1-c)]-b}{a b(1-c d)}, \frac{b[d+a(1-d)]-a}{a b(1-c d)}\right)$. One may look at System (5.2) as a perturbation of the one-dimensional logistic map $f(x)=\mu x(1-x)$, defined on $[0,1]$, where $0<\mu \leq 4$, whose dynamics is well-understood [6].

The domain of Model (5.2) is given by the following set

$$
\mathbf{D}=\left\{\left(x_{1}, x_{2}\right) \in \mathbb{R}^{2}: x_{1}+c x_{2} \leq 1 \wedge x_{2}+d x_{1} \leq 1 \wedge x_{1} \geq 0 \wedge x_{2} \geq 0\right\}
$$

In Fig. 1, we represent a prototype of the set $\mathbf{D}$. As is clearly seen we should assume that $0<c<1$ and $0<d<1$. Henceforth, we have the following assumption on the parameters concerning the new Cournot duopoly model

$$
0<a<4, \quad 0<b<4, \quad 0<c<1, \quad 0<d<1
$$

Notice that, in the domain $\mathbf{D}$ we have $x_{1} \leq 1$ and $x_{2} \leq 1$.

Let $F(x, y)=(a x(1-x-c y), b y(1-y-d x))$ be the map representing Model (5.2). Since the Jacobian of $F$ is given by

$$
J(x, y):=\left(\begin{array}{cc}
-2 x a-c y a+a & -a c x \\
-b d y & -d x b-2 y b+b
\end{array}\right)
$$


we have

$J(\mathbf{O})=\left(\begin{array}{ll}a & 0 \\ 0 & b\end{array}\right), \quad J\left(\mathbf{E}_{1}\right)=\left(\begin{array}{cc}2-a & c-a c \\ 0 & \frac{b(-d a+a+d)}{a}\end{array}\right), \quad J\left(\mathbf{E}_{2}\right)=\left(\begin{array}{cc}\frac{a(-c b+b+c)}{b} & 0 \\ d-b d & 2-b\end{array}\right)$,

and

$$
J\left(\mathbf{E}^{*}\right)=\left(\begin{array}{cc}
\frac{a c+b(-c a+a+c d-2)}{b(c d-1)} & -\frac{c(b(a(c-1)+1)-a c)}{b(c d-1)} \\
-\frac{d(a(b(d-1)+1)-b d)}{a(c d-1)} & \frac{b d+a(-d b+b+c d-2)}{a(c d-1)}
\end{array}\right)
$$

We will compute the region of local stability only for $\mathbf{E}^{*}$ since for the other fixed points it is trivial. For the matrix $J\left(\mathbf{E}^{*}\right)$ we have

$$
\Delta_{0}=\frac{-a^{2}(b(c-1)-c)(b(d-1)+2)+a b(b((c-3) d+2)-4)+2 b^{2} d}{a b(c d-1)}
$$

and

$$
\Delta_{1}=\frac{a^{2}(b(-c)+b+c)+a b(b(-d)+b+2 c d-4)+b^{2} d}{a b(c d-1)} .
$$

The following result gives conditions for local stability of the fixed points of Model (5.2) based in the necessary and sufficient conditions.

Proposition 5.1 Let $\mathbf{O}, \mathbf{E}_{1}, \mathbf{E}_{2}$ and $\mathbf{E}^{*}$ be the fixed points of Model (5.2) determined above such that Assumption (5.3) is satisfied. Then:

1. the fixed point $\mathbf{O}$ is locally asymptotically stable whenever $a \leq 1$ and $b \leq 1$;

2. the fixed point $\mathbf{E}_{1}$ is locally asymptotically stable whenever $1<a \leq 3$ and $b<\frac{a}{a(1-d)+d}$

3. the fixed point $\mathbf{E}_{2}$ is locally asymptotically stable whenever $1<b \leq 3$ and $a<\frac{b}{b(1-c)+c}$

4. the fixed point $\mathbf{E}^{*}$ is locally asymptotically stable whenever

$$
\begin{gathered}
-a^{2}(b(c-1)-c)(b(d-1)+3)+a b(b((c-4) d+3)+3 c d-9)+3 b^{2} d<0 \\
(b(a(c-1)+1)-a c)(a(b(d-1)+1)-b d)>0 \\
a^{2}(b(1-c)+c)+b^{2} d+\hat{a b}(-3+c d+b(1-d)) \geq 0
\end{gathered}
$$

A prototype of these stability regions, in the parameter space $a O b$, is depicted in Fig. 2 .

For similar studies in two-dimensional models, including the study of stability via the centre manifold, we refer the work by Luís et al. in [10] for the Ricker competition model and the work by Guzowska et al. in [7] for the logistic competition model. 
Fig. 2 Stability regions, in the parameter space $a O b$, of the fixed points of Model (5.2). In Region $\mathrm{O}$ the origin is locally asymptotically stable. In Region $\mathrm{R}$ (respectively Q) the exclusion fixed point $\mathbf{E}_{1}$ (respectively $\mathbf{E}_{2}$ ) is locally asymptotically stable. In Region $\mathrm{S}$ the positive fixed point $\mathbf{E}^{*}$ is locally asymptotically stable

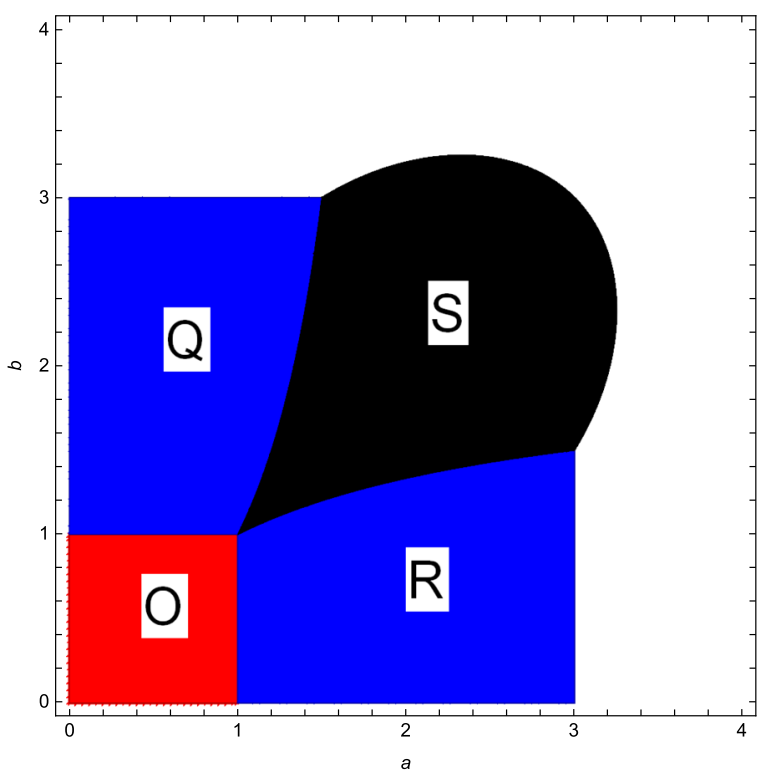

\subsection{Delay Logistic Model}

Let us consider the delay logistic equation given by $y_{n+1}=\lambda y_{n}\left(1-y_{n-(k-1)}\right), \lambda>0$ and $k \geq 2$ fixed. By letting $y_{n-i}=x_{i+1}(n), i=0,1, \ldots, k-1$, the dynamics of this equation is equivalent to dynamics of the $k$-dimensional the first-order system

$$
\left\{\begin{array}{l}
x_{1}(n+1)=\lambda x_{1}(n)\left(1-x_{k}(n)\right) \\
x_{2}(n+1)=x_{1}(n) \\
x_{3}(n+1)=x_{2}(n) \\
\vdots \\
x_{k}(n+1)=x_{k-1}(n)
\end{array}\right.
$$

Let $F\left(x_{1}, x_{2}, \ldots, x_{k}\right)=\left(\lambda x_{1}\left(1-x_{k}\right), x_{1}, \ldots, x_{k-1}\right)$ be the map representing System (5.4). The fixed points of $F$ are the origin $\mathbf{O}$ and $\mathbf{E}^{*}=\frac{\lambda-1}{\lambda}(1,1, \ldots, 1)$.

$$
J(\mathbf{O})=\left(\begin{array}{cc}
\lambda e_{1 \times(k-1)} & 0 \\
I_{k-1} & O_{(k-1) \times 1}
\end{array}\right), \quad J\left(\mathbf{E}^{*}\right)=\left(\begin{array}{cc}
e_{1 \times(k-1)} & 1-\lambda \\
I_{k-1} & O_{(k-1) \times 1}
\end{array}\right),
$$

where $I_{k-1}$ is the identity matrix, $e_{1 \times(k-1)}$ is the line matrix $\left(\begin{array}{lllll}1 & 0 & 0 & \ldots & 0\end{array}\right)$ and $O_{(k-1) \times 1}$ is a column null matrix.

For the matrix $J(\mathbf{O})$ we have $\Delta_{i}=0$, for $i=0, \ldots, k-2$ and $\Delta_{k-1}=\lambda$. Hence, the origin is locally asymptotically stable whenever $0<\lambda \leq 1$. Notice that, in the case of $\lambda=1$ it is necessary to use the center manifold theory for discrete dynamical systems. For general framework in this field we refer the books [5,6,9] and for specific results and terminology in discrete dynamical systems the papers $[7,10,12]$. 
Table 2 The right side of the interval of stability of the fixed point $\mathbf{E}^{*}$ of the delayed logistic model

\begin{tabular}{llll}
\hline$k$ & $\lambda_{k}$ & $k$ & $\lambda_{k}$ \\
\hline 2 & 2 & 8 & 1.2090569265353093 \\
3 & $\frac{1+\sqrt{5}}{2}$ & 9 & 1.184536718926269 \\
4 & 1.445041867912629 & 10 & 1.165158690928726 \\
5 & 1.3472963553338608 & 11 & 1.149460187247045 \\
6 & 1.2846296765465781 & 12 & 1.1364848266896812 \\
7 & 1.241073360510646 & $\ldots$ & $\ldots$ \\
\hline
\end{tabular}

Concerning the matrix $J\left(\mathbf{E}^{*}\right)$ we have $\Delta_{0}=(-1)^{k}(\lambda-1), \Delta_{i}=0$, for $i=$ $1, \ldots, k-2$ and $\Delta_{k-1}=1$.

We now study the necessary and sufficient conditions of local stability of the fixed point $\mathbf{E}^{*}$ accordingly to the dimension of the system (5.4).

When $k=2$, the necessary and sufficient conditions are equivalent to $0<\lambda<2$. Hence, the fixed point $\mathbf{E}^{*}$ is locally asymptotically stable whenever $0<\lambda<2=\lambda_{2}$.

When $k=3$, the necessary conditions is $|2-\lambda|<1$ and sufficient conditions are

$$
|1-\lambda|<1, \quad|\lambda(\lambda-2)|>|1-\lambda|
$$

This is equivalent to have $1<\lambda<\frac{1+\sqrt{5}}{2}=\lambda_{3}$.

For $k=4$ it follows that the necessary conditions is $\lambda>1$ and sufficient conditions are given by

$$
|1-\lambda|<1, \quad|\lambda(\lambda-2)|>|1-\lambda|, \quad\left|(\lambda(\lambda-2))^{2}-(\lambda-1)^{2}\right|>|\lambda-1| .
$$

These conditions are equivalent to $1<\lambda<\lambda_{4}$ (Table 2).

When $k=5$, the necessary conditions is equivalent to $|\lambda-2|<1$ and the sufficient conditions are given by

$$
\begin{aligned}
& |\lambda-1|<1, \quad|\lambda(\lambda-2)|>|1-\lambda|, \quad\left|\lambda^{2}(\lambda-2)^{2}-(1-\lambda)^{2}\right|>|\lambda-1| \\
& \left|\left(\lambda^{2}(\lambda-2)^{2}-(1-\lambda)^{2}\right)^{2}-(\lambda-1)^{2}\right|>|\lambda(\lambda-1)(\lambda-2)| .
\end{aligned}
$$

Simplifying, the necessary and sufficient conditions are equivalent to $1<\lambda<\lambda_{5}$, i.e., the fixed point $\mathbf{E}^{*}$ is locally asymptotically stable whenever $1<\lambda<\lambda_{5}$.

Following the previous computations, we are able to determine the sequence of values of $\lambda_{k}$, given in Table 2. We summarize in the next result the previous ideas.

Proposition 5.2 Let $\mathbf{O}$ and $\mathbf{E}^{*}$ be the fixed points of the $k$-dimensional first-order system (5.4). Then

1. the fixed point $\mathbf{O}$ is locally asymptotically stable whenever $0<\lambda \leq 1$.

2. the fixed point $\mathbf{E}^{*}$ is locally asymptotically stable whenever $1<\lambda<\lambda_{k}$, where $\lambda_{k}$ are given in Table 2 for $k=2, \ldots, 12$. 


\subsection{Ricker Competition Model of 4 Species}

The 4-dimensional Ricker competition model [13, page 31] is usually written as

$$
\left\{\begin{array}{l}
x_{1}(n+1)=x_{1}(n) \exp \left(r_{1}-\sum_{i=1}^{4} c_{1 i} x_{i}(n)\right) \\
x_{2}(n+1)=x_{2}(n) \exp \left(r_{2}-\sum_{i=1}^{4} c_{2 i} x_{i}(n)\right) \\
x_{3}(n+1)=x_{3}(n) \exp \left(r_{3}-\sum_{i=1}^{4} c_{3 i} x_{i}(n)\right) \\
x_{4}(n+1)=x_{4}(n) \exp \left(r_{4}-\sum_{i=1}^{4} c_{4 i} x_{i}(n)\right)
\end{array}\right.
$$

where $x_{i}(n)$, for $i=1,2,3,4$, represents the population sizes of species $x_{i}$ at time unit $n$, the parameters $r_{i}$, for $i=1,2,3,4$, are the inherent exponential growth rates at low densities and $c_{i, j}$, for $i, j=1,2,3,4$, are the competition intensity coefficients measuring the effects of intra-specific competition and inter-specific competition. More precisely, $c_{i i}$ are the intra-specific competition parameters, while $c_{i j}$, for $i \neq j$ are the inter-specific competition parameters. Notice that, these 20 parameters are assumed to be positive. For a complete study in local dynamics of this model in dimensions 1 , 2 and 3 we refer the papers $[10,11]$.

For the sake of simplicity, we assume that the intra-specific competition parameters are equal to one, i.e, $c_{i i}=1$, and the inter-specific competition parameters lie on the unit interval, i.e., $0<c_{i j}<1$, for $i \neq j$. Moreover, in order to make the algebraic manipulations feasible we assume that $c_{i j}=c$, for $i \neq j$, i.e, we study the model in the symmetric case.

Let $F=\left(f_{1}, f_{2}, f_{3}, f_{4}\right)$ be the map representing System (5.7), where $f_{i}\left(x_{1}, x_{2}, x_{3}\right.$, $\left.x_{4}\right)$ are given by

$$
\begin{aligned}
& f_{1}\left(x_{1}, x_{2}, x_{3}, x_{4}\right)=x_{1} e^{r_{1}-x_{1}-c\left(x_{2}+x_{3}+x_{4}\right)}, \quad f_{2}\left(x_{1}, x_{2}, x_{3}, x_{4}\right)=x_{2} e^{r_{2}-x_{2}-c\left(x_{1}+x_{3}+x_{4}\right)} \\
& f_{3}\left(x_{1}, x_{2}, x_{3}, x_{4}\right)=x_{3} e^{r_{3}-x_{3}-c\left(x_{1}+x_{2}+x_{4}\right)}, \quad f_{4}\left(x_{1}, x_{2}, x_{3}, x_{4}\right)=x_{4} e^{r_{4}-x_{4}-c\left(x_{1}+x_{2}+x_{3}\right)} .
\end{aligned}
$$

The map $F$ has sixteen fixed points, namely the origin $\mathbf{O}$, four fixed points on the axes

$$
\mathbf{E}_{\mathbf{1}}=\left(r_{1}, 0,0,0\right), \quad \mathbf{E}_{\mathbf{2}}=\left(0, r_{2}, 0,0\right), \quad \mathbf{E}_{\mathbf{3}}=\left(0,0, r_{3}, 0\right), \quad \mathbf{E}_{\mathbf{4}}=\left(0,0,0, r_{4}\right),
$$

six fixed points in the coordinate planes

$$
\begin{aligned}
& \mathbf{E}_{\mathbf{1 2}}=\left(\frac{r_{1}-c r_{2}}{1-c^{2}}, \frac{r_{2}-c r_{1}}{1-c^{2}}, 0,0\right), \quad \mathbf{E}_{\mathbf{1 3}}=\left(\frac{r_{1}-c r_{3}}{1-c^{2}}, 0, \frac{r_{3}-c r_{1}}{1-c^{2}}, 0\right), \\
& \mathbf{E}_{\mathbf{1 4}}=\left(\frac{r_{1}-c r_{4}}{1-c^{2}}, 0,0, \frac{r_{4}-c r_{1}}{1-c^{2}}\right), \quad \mathbf{E}_{\mathbf{2 3}}=\left(0, \frac{r_{2}-c r_{3}}{1-c^{2}}, \frac{r_{3}-c r_{2}}{1-c^{2}}, 0\right), \\
& \mathbf{E}_{\mathbf{2 4}}=\left(0, \frac{r_{2}-c r_{4}}{1-c^{2}}, 0, \frac{r_{4}-c r_{2}}{1-c^{2}}\right), \quad \mathbf{E}_{\mathbf{3 4}}=\left(0,0, \frac{r_{3}-c r_{4}}{1-c^{2}}, \frac{r_{4}-c r_{3}}{1-c^{2}}\right),
\end{aligned}
$$

four fixed points in the coordinate surfaces

$$
\mathbf{E}_{\mathbf{1 2 3}}=\left(\frac{(c+1) r_{1}-c\left(r_{2}+r_{3}\right)}{(1-c)(2 c+1)}, \frac{(c+1) r_{2}-c\left(r_{1}+r_{3}\right)}{(1-c)(2 c+1)}, \frac{(c+1) r_{3}-c\left(r_{1}+r_{2}\right)}{(1-c)(2 c+1)}, 0\right),
$$


$\mathbf{E}_{\mathbf{1 2 4}}=\left(\frac{(c+1) r_{1}-c\left(r_{2}+r_{4}\right)}{(1-c)(2 c+1)}, \frac{(c+1) r_{2}-c\left(r_{1}+r_{4}\right)}{(1-c)(2 c+1)}, 0, \frac{(c+1) r_{4}-c\left(r_{1}+r_{2}\right)}{(1-c)(2 c+1)}\right)$,
$\mathbf{E}_{\mathbf{1 3 4}}=\left(\frac{(c+1) r_{1}-c\left(r_{3}+r_{4}\right)}{(1-c)(2 c+1)}, 0, \frac{(c+1) r_{3}-c\left(r_{1}+r_{4}\right)}{(1-c)(2 c+1)}, \frac{(c+1) r_{4}-c\left(r_{1}+r_{3}\right)}{(1-c)(2 c+1)}\right)$,
$\mathbf{E}_{\mathbf{2 3 4}}=\left(0, \frac{(c+1) r_{2}-c\left(r_{3}+r_{4}\right)}{(1-c)(2 c+1)}, \frac{(c+1) r_{3}-c\left(r_{2}+r_{4}\right)}{(1-c)(2 c+1)}, \frac{(c+1) r_{4}-c\left(r_{2}+r_{3}\right)}{(1-c)(2 c+1)}\right)$,

and a possible interior fixed point given by

$$
\begin{aligned}
\mathbf{E}^{*}= & \left(\frac{(2 c+1) r_{1}-c\left(r_{2}+r_{3}+r_{4}\right)}{(1-c)(3 c+1)}, \frac{(2 c+1) r_{2}-c\left(r_{1}+r_{3}+r_{4}\right)}{(1-c)(3 c+1)}\right. \\
& \left.\frac{(2 c+1) r_{3}-c\left(r_{1}+r_{2}+r_{4}\right)}{(1-c)(3 c+1)}, \frac{(2 c+1) r_{4}-c\left(r_{1}+r_{2}+r_{3}\right)}{(1-c)(3 c+1)}\right) .
\end{aligned}
$$

Since the main purpose of the model is population dynamics, we always assume that those fixed points are non-negative.

The origin is always unstable since the eigenvalues of $J(\mathbf{O})$ are $e^{r_{i}}>0, i=$ $1, \ldots, 4$. The fixed point $\mathbf{E}_{\mathbf{i}}$ is locally asymptotically stable whenever

$$
0<r_{i}<2 \text { and } r_{j}<c r_{i}, \quad j \neq i, \quad i, j=1, \ldots, 4 \text {. }
$$

Concerning the matrix $J\left(\mathbf{E}_{\mathbf{1 2}}\right)$ (the study of the other fixed points on the axes follows in a similar way) we have $\Delta_{0}=\frac{c-\psi}{c}+\omega$

$$
\begin{aligned}
& \Delta_{1}=e^{r_{3}+r_{4}-2 \psi}+\frac{c-\psi}{c}\left(e^{r_{3}}+e^{r_{4}}+e^{r_{3}+r_{4}-\psi}\right) e^{-\psi}+\omega e^{-\psi}\left(e^{r_{3}}+e^{r_{4}}\right), \\
& \Delta_{2}=e^{r_{3}+r_{4}-2 \psi}-1+\frac{2 c-\psi}{c}\left(e^{\psi}+e^{r_{3}}+e^{r_{4}}\right) e^{-\psi}+\omega,
\end{aligned}
$$

and

$$
\Delta_{3}=\left(2 e^{\psi}+e^{r_{3}}+e^{r_{4}}\right) e^{-\psi}-\frac{\psi}{c},
$$

where $\psi=\frac{c\left(r_{1}+r_{2}\right)}{c+1}$ and $\omega=\frac{\left(1+c^{2}\right) r_{1} r_{2}-c\left(r_{1}^{2}+r_{2}^{2}\right)}{1-c^{2}}$.

At this point, the algebraic manipulations are complicated due the number of parameters. Hence, we are not able to determine analytically the necessary and sufficient conditions of stability for all values of the parameters. However, since $e^{r_{i}-\psi}, i=3,4$ are two eigenvalues of $J\left(\mathbf{E}_{\mathbf{1 2}}\right)$, in order to have local stability we need to have $r_{i}<\psi$, $i=3$, 4. Hence, considering $r_{3}=0.6$ and $r_{4}=0.5$ for $c=0.5$, we depict in Fig. 3 the stability region of the fixed point $\mathbf{E}_{\mathbf{1 2}}$ in the parameter space $r_{1} O r_{2}$, i.e., the region where the necessary and sufficient conditions are satisfied.

Now, concerning the fixed point $\mathbf{E}_{\mathbf{1 2 3}}$ one can follow the same techniques as before and find the region of local stability. After some algebraic manipulations in a software such as Mathematica, we depict in Fig. 4 this region, in the parameter space $r_{1} \mathrm{Or}_{2} \mathrm{Or}_{3}$, when $r_{4}=0.5$ and $c=0.5$. 
Fig. 3 Stability region, in the parameter space $r_{1} O r_{2}$, of the fixed point $\mathbf{E}_{12}$ of the model (5.7) when $c=0.5, r_{3}=0.6$ and $r_{4}=0.5$

Fig. 4 Stability region, in the parameter space $r_{1} \mathrm{Or}_{2} \mathrm{Or}_{3}$, of the fixed point $\mathbf{E}_{\mathbf{1 2 3}}$ of the model (5.7) when $c=0.5$ and $r_{4}=0.5$
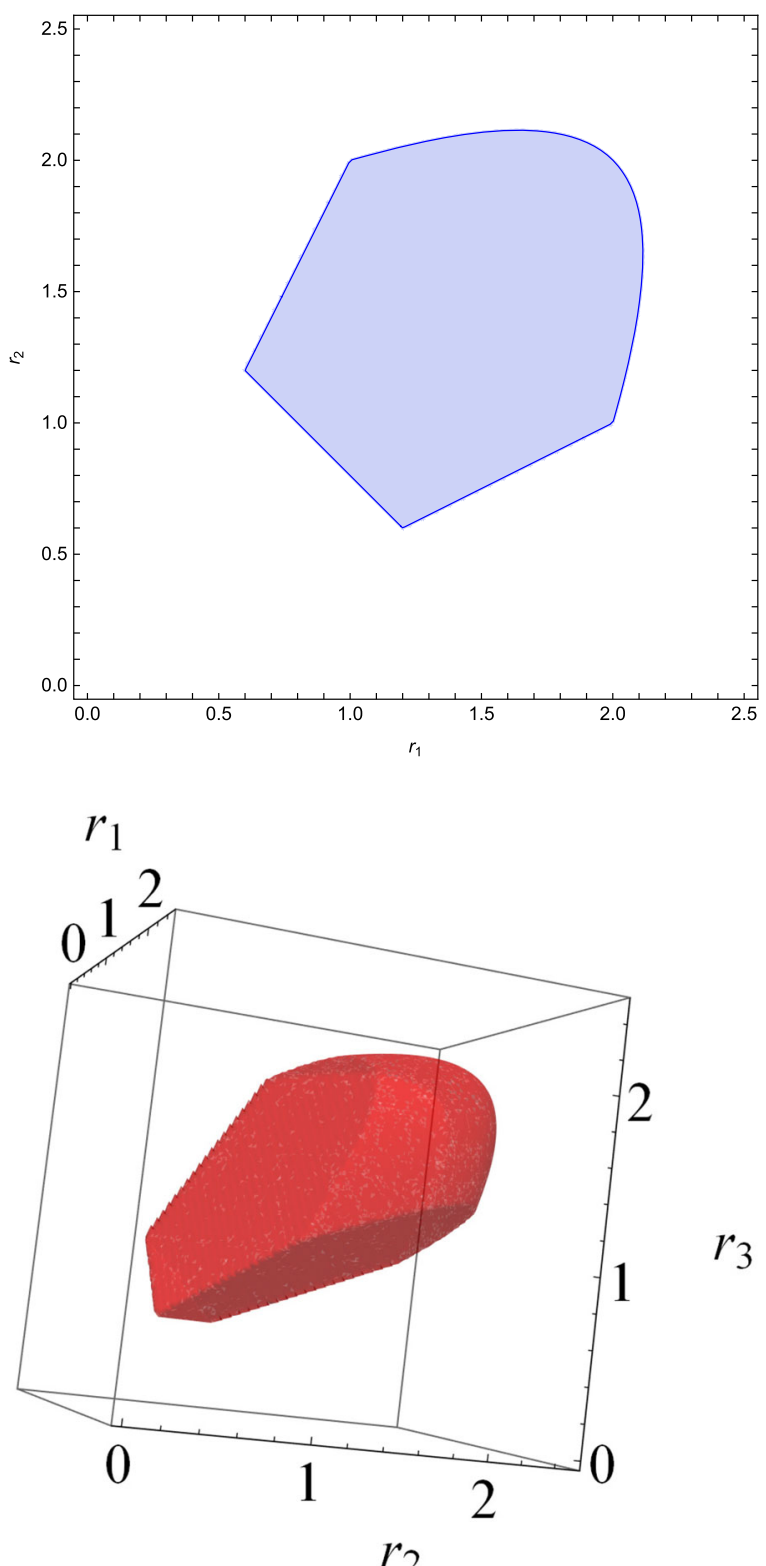

$r_{2}$

It remains to study the stability of the interior fixed point $\mathbf{E}^{*}$. Since the computations are long if we keep all the parameters, as it is clear in the previous cases, we will study a particular case. Let $c=0.5, r_{3}=1.6$ and $r_{4}=1.5$. The interior fixed point is given by

$$
\mathbf{E}^{*}=\left(1.6 r_{1}-0.4 r_{2}-1.24,-0.4 r_{1}+1.6 r_{2}-1.24,-0.4 r_{1}-0.4 r_{2}+1.96,\right.
$$


Fig. 5 Domain, in the parameter space $r_{1} O r_{2}$, where the fixed point $\mathbf{E}^{*}$ is positive when $c=0.5, r_{3}=1.6$ and $r_{4}=1.5$

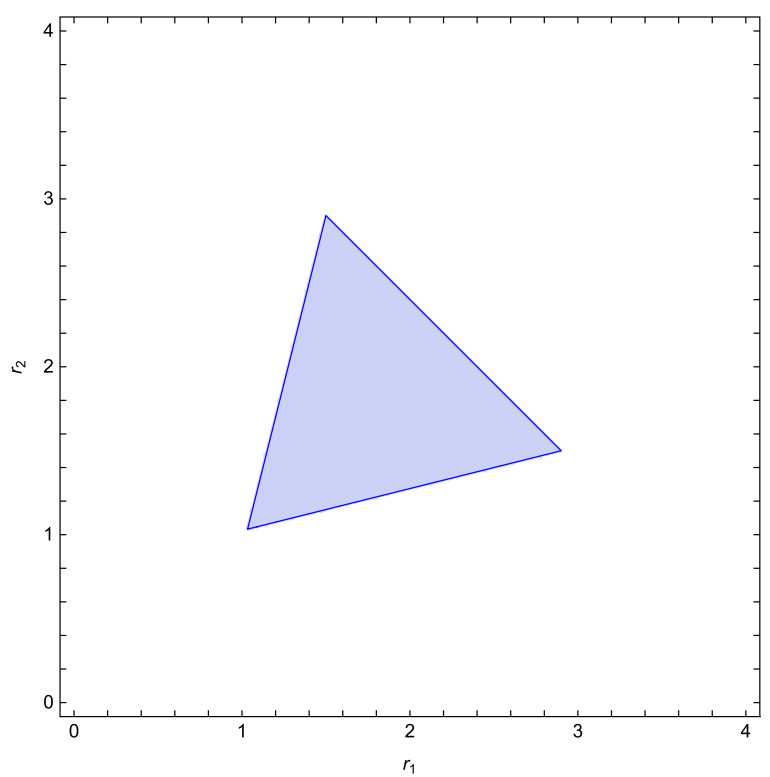

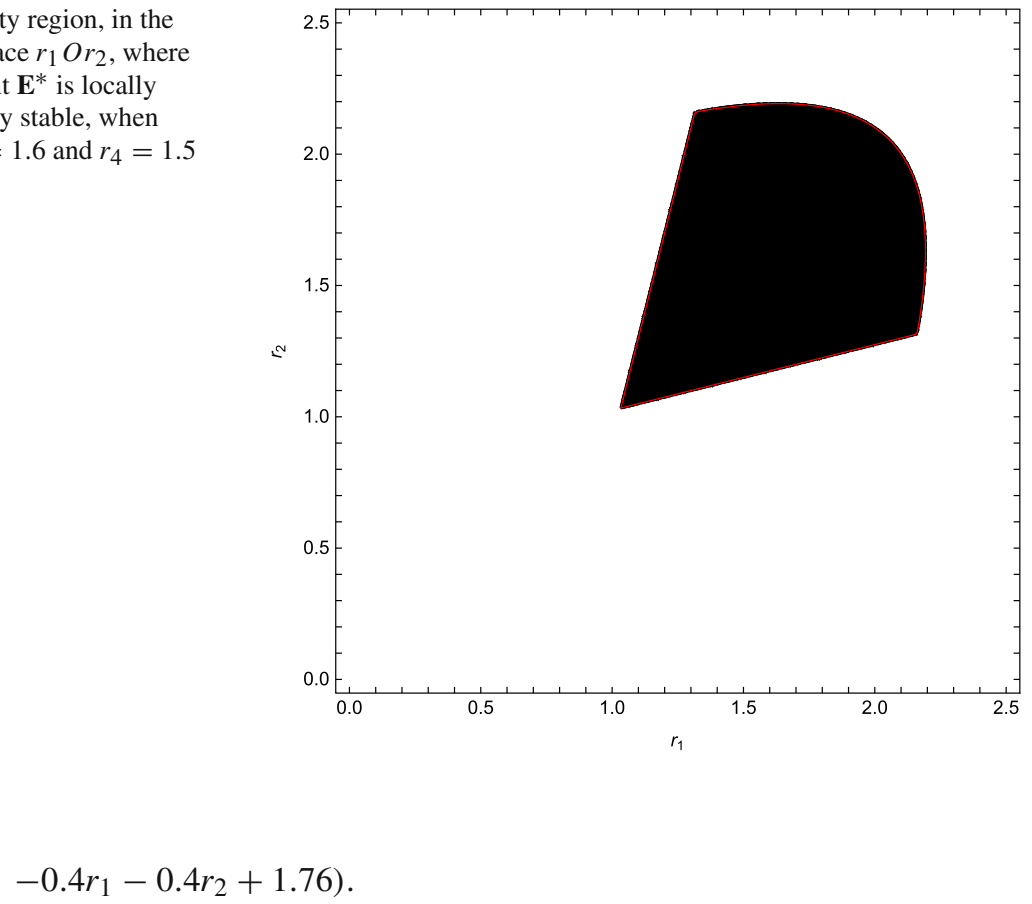

Fig. 6 Stability region, in the parameter space $r_{1} O r_{2}$, where the fixed point $\mathbf{E}^{*}$ is locally asymptotically stable, when $c=0.5, r_{3}=1.6$ and $r_{4}=1.5$

It is clear that we have to insure that $\mathbf{E}^{*}$ is positive. This occurs when $r_{1}$ and $r_{2}$ are in the region depicted in Fig. 5. 
Fig. 7 Relative position between the stability region of $\mathbf{E}^{*}$ and the region where $\mathbf{E}^{*}$ is positive

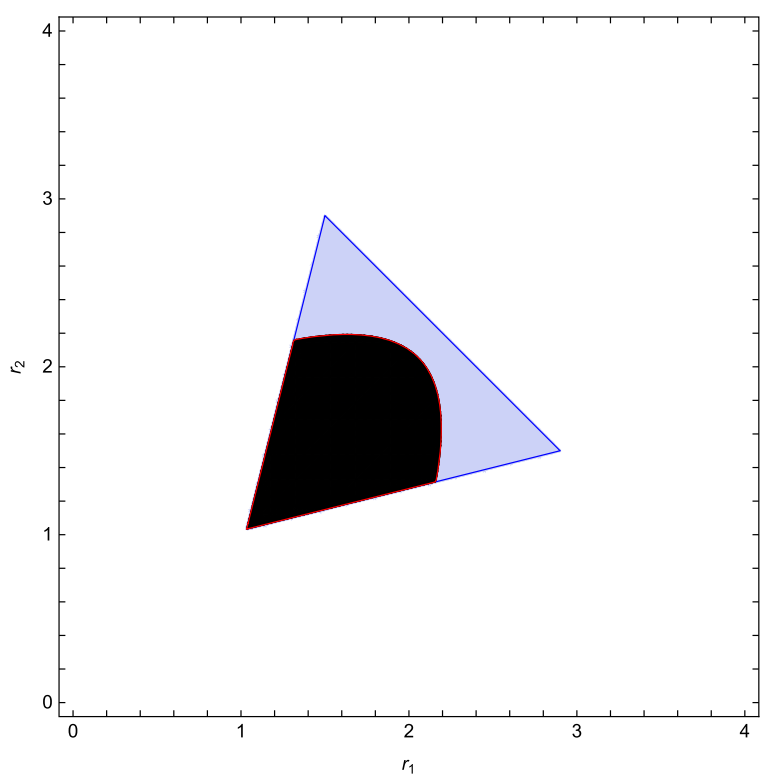

The Jacobian matrix $J\left(\mathbf{E}^{*}\right)$ is given by

$$
\left(\begin{array}{cccc}
-1.6 r_{1}+0.4 r_{2}+2.24 & -0.8 r_{1}+0.2 r_{2}+0.62 & -0.8 r_{1}+0.2 r_{2}+0.62 & -0.8 r_{1}+0.2 r_{2}+0.62 \\
0.2 r_{1}-0.8 r_{2}+0.62 & 0.4 r_{1}-1.6 r_{2}+2.24 & 0.2 r_{1}-0.8 r_{2}+0.62 & 0.2 r_{1}-0.8 r_{2}+0.62 \\
0.2 r_{1}+0.2 r_{2}-0.98 & 0.2 r_{1}+0.2 r_{2}-0.98 & 0.4 r_{1}+0.4 r_{2}-0.96 & 0.2 r_{1}+0.2 r_{2}-0.98 \\
0.2 r_{1}+0.2 r_{2}-0.88 & 0.2 r_{1}+0.2 r_{2}-0.88 & 0.2 r_{1}+0.2 r_{2}-0.88 & 0.4 r_{1}+0.4 r_{2}-0.76
\end{array}\right)
$$

The necessary and sufficient conditions are satisfied whenever the parameters belong in the region depicted in Fig. 6, i.e, the region in the parameter space $r_{1} \mathrm{Or}_{2}$ where the interior fixed point is locally stable, in this case.

Notice that, the region depicted in Fig. 6 is contained in the region depicted in Fig. 5, as it is illustrated in Fig. 7.

Acknowledgements This work was partially supported by FCT/Portugal through the Project UIDB/04459/2020.

\section{References}

1. Bischi, G., Stefanini, L., Gardini, L.: Synchronization, intermittency and critical curves in a duopoly game. Math. Comput. Simul. 44, 559-585 (1998)

2. Brooks, B.P.: Linear stability conditions for a first-order three-dimensional discrete dynamic. Appl. Math. Lett. 17(4), 463-466 (2004)

3. Brooks, B.P.: The coefficients of the characteristic polynomial in terms of the eigenvalues and the elements of an $n \times n$ matrix. Appl. Math. Lett. 19(6), 511-515 (2006)

4. Brooks, B.P.: Linear stability conditions for a first order 4-dimensional discrete dynamic. Appl. Comput. Math. 3(5), 463 (2014)

5. Carr, J.: Applications of Center Manifold Theory. Springer, New York (1982) 
6. Elaydi, S.: Discrete Chaos: With Applications in Science and Engineering, 2nd edn. Chapman \& Hall/CRC, Boca Raton (2007)

7. Guzowska, M., Luís, R., Elaydi, S.: Bifurcation and invariant manifolds of the logistic competition model. J. Differ. Equ. Appl. 17(12), 1851-1872 (2011)

8. Jury, E.I.: On the roots of a real polynomial inside the unit circle and a stability criterion for linear discrete systems. In: IFAC Proceedings: 2nd International IFAC Congress on Automatic and Remote Control: Theory, Basle, Switzerland, Vol. 1(2), pp. 142-153 (1963)

9. Kuznetsov, Y.A.: Elements of Applied Bifurcation Theory. Applied Mathematical Sciences, vol. 112, 3rd edn. Springer, Berlin (2004)

10. Luís, R., Elaydi, S., Oliveira, H.: Stability of a Ricker-type competition model and the competitive exclusion principle. J. Biol. Dyn. 5(6), 636-660 (2011)

11. Luís, R., Rodrigues, E.: Local stability in 3D discrete dynamical systems: application to a Ricker competition model. Discrete Dyn. Nat Soc. 2017 (2017)

12. Psarros, N., Papaschinopoulos, G., Schinas, C.J.: Study of the stability of a system of difference equations using centre manifold theory. Appl. Math. Lett. 64, 185-192 (2017)

13. Ricker, E.: Handbook of computation for biological statistics of fish populations. Bulletin 119 of the Fisheries Resource Board Canada (1958)

14. Routh, E.J.: A Treatise on the Stability of a Given State of Motion: Particularly Steady Motion. Macmillan, London (1877)

Publisher's Note Springer Nature remains neutral with regard to jurisdictional claims in published maps and institutional affiliations. 